\title{
A CASE STUDY ON THROUGH-WATER DENSE IMAGE MATCHING
}

\author{
G. Mandlburger ${ }^{\mathrm{a}, \mathrm{b}}$ \\ ${ }^{a}$ University of Stuttgart, Institute for Photogrammetry, Stuttgart, Germany - gottfried.mandlburger@ifp.uni-stuttgart.de \\ ${ }^{\mathrm{b}}$ TU Vienna, Department of Geodesy and Geoinformation, Vienna, Austria - gottfried.mandlburger@geo.tuwien.ac.at
}

\author{
Commission II, WG II/9
}

\begin{abstract}
KEY WORDS: Multi-media photogrammetry, semi global matching, bathymetry, hydrography, shallow water mapping, dense point
\end{abstract} clouds, multi-spectral images

\begin{abstract}
:
In the last years, the tremendous progress in image processing and camera technology has reactivated the interest in photogrammetrybased surface mapping. With the advent of Dense Image Matching (DIM), the derivation of height values on a per-pixel basis became feasible, allowing the derivation of Digital Elevation Models (DEM) with a spatial resolution in the range of the ground sampling distance of the aerial images, which is often below $10 \mathrm{~cm}$ today. While mapping topography and vegetation constitutes the primary field of application for image based surface reconstruction, multi-spectral images also allow to see through the water surface to the bottom underneath provided sufficient water clarity. In this contribution, the feasibility of through-water dense image matching for mapping shallow water bathymetry using off-the-shelf software is evaluated. In a case study, the SURE software is applied to three different coastal and inland water bodies. After refraction correction, the DIM point clouds and the DEMs derived thereof are compared to concurrently acquired laser bathymetry data. The results confirm the general suitability of through-water dense image matching, but sufficient bottom texture and favorable environmental conditions (clear water, calm water surface) are a preconditions for achieving accurate results. Water depths of up to $5 \mathrm{~m}$ could be mapped with a mean deviation between laser and trough-water DIM in the $\mathrm{dm}$-range. Image based water depth estimates, however, become unreliable in case of turbid or wavy water and poor bottom texture.
\end{abstract}

\section{INTRODUCTION}

Although topo-bathymetric LiDAR (Light Detection And Ranging) has become the state-of-the art for mapping shallow coastal and inland water bodies, most modern sensors provide high resolution multi-spectral cameras along with the LiDAR instruments. While the multi-spectral image content can directly be used to estimate water depths by establishing a color-to-depth relation based on physical and/or regression models (Lyzenga et al., 2006; Legleiter, 2016), the advent of Dense Image Matching (DOM, Hirschmuller (2008)) has revived the interest in through-water photogrammetry (Westaway et al., 2001) for charting sufficiently transparent water bodies with applications in archeology, ecology, hydraulics and the like. Despite the accuracy limitations of the photogrammetric approach depending on water depth, water surface planarity, water clarity, etc. (Maas, 2015), the availability of imagery with Ground Sampling Distances (GSD) less than $10 \mathrm{~cm}$ fuels the hope for high resolution mapping of the shallow water-land-transition zone.

The main problem in both through-water photogrammetry and LiDAR bathymetry is the refraction at the air-water interface causing a change of the direction of the image ray (Maas, 2015) as well as a change of the propagation velocity of the laser pulse (Birkebak et al., 2018). In both cases knowledge of exact water level heights are a precondition for refraction and run-time correction of the raw measurements. The advantage of bathymetric LiDAR over multi-media photogrammetry is hereby two-fold: (i) LiDAR is a polar data acquisition strategy, meaning that a single measurement is sufficient to obtain a $3 \mathrm{D}$ point and (ii) the employed green laser light interacts with the water surface, the water column, and the water bottom and, thus, simultaneously delivers height estimates of the surface and the bottom (Guenther et al., 2000; Schwarz et al., 2017). While image based water level estimation remains a challenging task due to the specular and dynamic surface (Rupnik et al., 2015), especially the infrared channel of multi-spectral images can be used to detect the waterland-boundary from which the water level can be derived at least for calm water surfaces.

Apart from that, image ray bending at the air-water interface also causes a violation of the collinearity condition constituting the inherent mathematical model of most photogrammetric software, and image blurring due to multi-directional scattering within the water column further hampers image matching. Against this background, this contribution evaluates the applicability of multimedia DIM for mapping shallow water bathymetry based on offthe-shelf software and processing pipelines for different test sites. The study areas comprise coastal scenes (Malaysian coast, German Baltic Sea) and an alpine mountain lake (Stubai valley, Tyrol, Austria). Being aware of the accuracy restrictions reported in literature (Maas, 2015), the presented case study aims to answer the question under which conditions reliable results can be expected under real-world conditions. This will be addressed by comparing the photogrammetrically derived depth estimates with airborne LiDAR data as reference.

The remainder of this article is structured as follows: In Section 2 the study areas are introduced and the general processing strategies are detailed. The achieved results are presented in Section 3 for each test site separately and the outcomes are discussed qualitatively by visual inspection and quantitatively by comparing the through-water DIM point clouds and the derived Digital Elevation Models (DEM) with concurrently acquired topo-bathymetric LiDAR data. The main findings are summarized in Section 4 together with an outlook on future research on subject matters. 


\begin{tabular}{|c|c|c|c|c|c|c|c|c|}
\hline Area & Site & Year & $\begin{array}{r}\text { Location } \\
\text { North } \\
\end{array}$ & East & LiDAR & Camera & $\begin{array}{r}\text { Sensor dimension } \\
\text { [pixel x pixel] }\end{array}$ & $\begin{array}{l}\text { GSD } \\
{[\mathrm{cm}]}\end{array}$ \\
\hline I & Malaysia & 2017 & $5^{\circ} 57^{\prime}$ & $116^{\circ} 01$ & - & UltraCam Osprey & $11674 \times 7514$ & 7 \\
\hline II & Poel, Germany & 2014 & $54^{\circ} 03$ & $11^{\circ} 31^{\prime}$ & HawkEye III & $\mathrm{RCD} 30$ & $9000 \times 6732$ & 6 \\
\hline III & Stubai valley, Austria & 2017 & $47^{\circ} 00^{\prime}$ & $11^{\circ} 11^{\prime}$ & VQ-880-G & Hasselblad H39 & $7216 \times 5412$ & 10 \\
\hline & & 2016 & & & 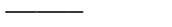 & UltraCamXp & $17310 \times 11310$ & 16 \\
\hline
\end{tabular}

Table 1. Study areas and sensor characteristics

\section{MATERIALS AND METHODS}

In this section the study areas are introduced (Section 2.1) and the applied data processing strategies and evaluation methods are presented (Section 2.2).

\subsection{Study area}

For the study at hand, data from three test sites were available. The locations of the study areas and the respective data capturing details are summarized in Table1. Area I (Borneo, Malaysia) comprises an in-shore harbor and the surrounding residential area as well as a wooded coastal section with near-shore reef structures, but only the latter was examined. Area II (Poel, Baltic Sea, Germany, cf. Song et al. (2015)) features bright sandy soil to both sides of a small peninsula with embedded patches of dark sea grass (cf. Figure 2). Area III (Blaue Lacke, Stubai valley, Tyrol, Austria) is a high mountain lake with a bed rock surface, featuring boulders of different sizes in the littoral area to both sides of the waterline. Multi-temporal data is available for this site (2017: LiDAR + RGB imagery, 2016: RGBI imagery) but only the prior was examined in detail.

\subsection{Data processing}

For each site the following processing steps were carried out:

- image pre-processing and conversion to 16-bit TIFF using the respective camera software

- image bundle block adjustment using standard software (Pix4D: Pix4D mapper, Trimble: Match-AT, Leica: Oriama and IPAS CO+, Vexcel: UltraMap)

- laser scanning strip adjustment and co-registration of scans and images (Glira et al., 2015; Mandlburger et al., 2017) using the scientific laser scanning software OPALS (Pfeifer et al., 2014)

- dense image matching using SURE (Rothermel et al., 2012; Wenzel et al., 2013)

- extraction of a water surface model from the laser data

- refraction correction of the raw submerged DIM points (Wimmer, 2016)

- data post-processing and comparison of LiDAR and image derived bathymetry.

Proper image orientation via bundle block adjustment (including estimation of the interior orientation, image distortion, and exterior orientation) is a precondition for high quality surface reconstruction via DIM. The mountain lake dataset (Blaue Lacke, Area III) was hereby small enough so that enough image tie points in the bare earth area around the lake (not affected by refraction effects) were available to properly connect the image block. Flight lines in higher altitudes further helped to stabilize the entire image block. The respective re-projection error for Area III was 0.12 pixels and the RMSE at 20 control points measured with dGPS amounted to $2 \mathrm{~cm}$ in planimetry and $4 \mathrm{~cm}$ in elevation. For the coastal datasets, a different strategy was necessary as a predominant part of the aerial images contain water areas only. In this case, the data providers' bundle block adjustment software (Area I: Vexcel: UltraMap, Area II: Oriama, IPAS CO+) was employed to estimate the inner and outer orientation of the cameras as well as the mounting calibration parameters (camera lever arms and boresight angles) based on the dry land parts of the flight blocks. The final image orientations were subsequently derived by combining the calibrated lever arms and boresight angles with the flight trajectory data calculated from Global Navigation Satellite System (GNSS) and Inertial Measurement Unit (IMU) observations. In this case, the quality of the orientation data was checked by analyzing the DIM results obtained from stereo models on dry ground. As no layering effects could be observed, the image orientations were considered adequate for the purpose of through-water dense matching.

For the test areas featuring concurrently acquired laser and image data (i.e. Area II and III), the registration precision of the LiDAR and DIM point clouds was assessed by deriving DEM rasters with a grid spacing of $25 \mathrm{~cm}$ for both data sources independently and by evaluating the height discrepancies. In all cases, the vertical deviations calculated for smooth dry land areas was below $3 \mathrm{~cm}$ confirming a good relative orientation of the image and LiDAR derived point clouds.

Dense matching was carried out for all stereo models separately (i.e. no multi-view stereo triangulation) in order to guarantee proper refraction correction of the resulting 3D points according to Snells law (Maas, 2015; Wimmer, 2016). The apparent image rays were hereby intersected with the water surface model derived either from LiDAR (Area II and III) or by assuming a horizontal water level with the height derived from the water-land-boundary (Area I). As the forward image overlap was at least $60 \%$ for all datasets, stereo models were built based on pairs of neighboring images and pairs with one but the next image.

The post-processing steps comprised refraction correction of the submerged laser points (Mandlburger et al., 2015) and DIM points (Wimmer, 2016), the calculation of relative measures for the DIM point clouds (local roughness) at land and within the water domain, DEM interpolation including smoothing and filtering of measurement errors, and absolute comparison of LiDAR and image derived underwater DEMs. Whereas moving least squares interpolation was used for the LiDAR data, a median based filtering was applied for the DIM point clouds. Depending on the point density, in a first step a $25 \mathrm{~cm}$ raster was calculated using the median elevation of all cell points as representative cell value. In most cases further smoothing (median filtering in a $1 \mathrm{~m}$ kernel area) was necessary to obtain acceptable results, thus, reducing the effective DIM DEM resolution. 

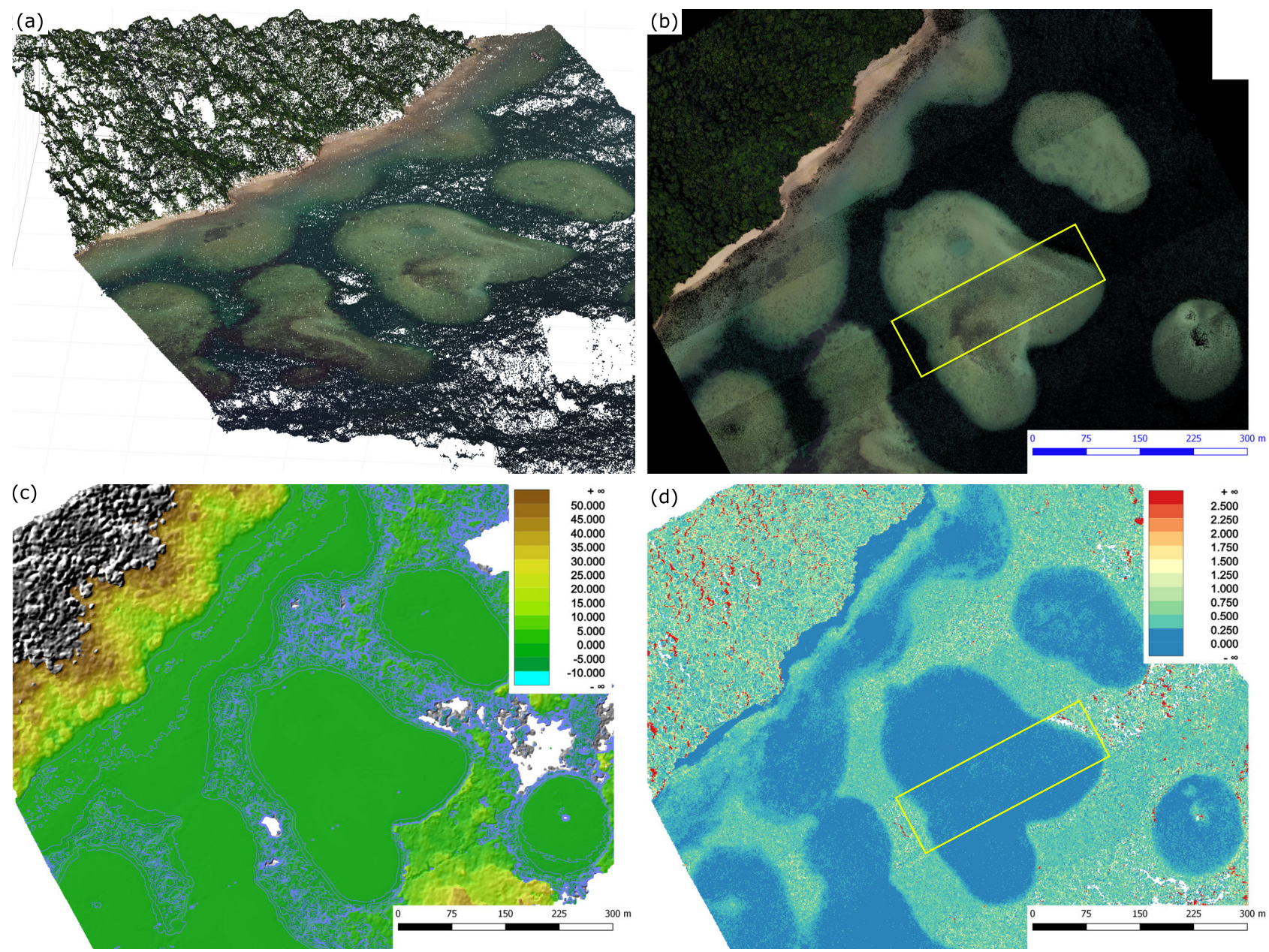

Figure 1. Selected Dense Image Matching results for Area I (Borneo, Malaysia); (a) Perspective view of colorized 3D DEM point cloud; (b) Digital RGB Orthophoto; (c) DEM raster (grid spacing: 50cm), Shaded relief map superimposed with color coded elevation map; (d) Roughness map (RMSE of DIM point cloud, analysis unit: $25 \times 25 \mathrm{~cm}^{2}$ ).

\section{RESULTS AND DISCUSSIONS}

In this section, through-water DIM results are presented and discussed. The accuracy of the achieved DIM DEMs is assessed quantitatively based on the topo-bathymetric LiDAR data as reference. Using LiDAR as the comparison basis is justified, as the technique is well established for mapping shallow water areas (Guenther et al., 2000; Mandlburger et al., 2015) and all used sensors (Area II: HaykEye III, Area III: VQ-880-G) satisfy the most rigorous hydrographic survey standards published by the International Hydrographic Organization (IHO) requesting a Total Vertical Uncertainty (TVU) of less than $25 \mathrm{~cm}$ (IHO, 2008). The latter specifically applies to those areas providing enough water transparency to allow depth estimation via through-water photogrammetry. Wherever no LiDAR data was available (Area I), the results are evaluated qualitatively by visual inspection.

\subsection{Area I}

Selected DIM results for Area I (Borneo, Malaysia) are displayed in Figure 1. The general feasibility to derive shallow under-water topography with through-water DIM is demonstrated by the perspective view of the DIM-derived DEM points of Figure 1a. The scene covers a sandy beach area with a steep wooded slope on the north-western landward side of the scene. The submerged shallow water part features large patches of bright sand with embedded reef structures providing good image texture and, thus, good conditions for image matching. The area between the sandy patches, in turn, is covered with sea grass and appears very dark (cf. orthophoto mosaic, Figure 1b).

It can clearly be seen from Figure 1a that through-water DIM works best in the well-textured bright sand areas, where the displayed DEM points appear both smooth and dense. While the higher DEM volatility in the north-western (dry) part of the scene can be explained by the steepness of the slope and the undulation of the tree canopy, the submerged dark area points are incomplete and, if present, the heights are rather unreliable. The latter especially applies to the deeper areas towards the south-eastern border of the scene, where the DEM rises above the water level indicated by the yellowish and brownish color tones visible in the color coded DEM height map (Figure 1c). In this figure, the colored DEM heights are superimposed with the shaded relief map and $1 \mathrm{~m}$-contour lines. The bright sandy areas also appear smooth in the DEM map whereas a more chaotic behavior in the dark sea grass area between the sandy patches is evident from the density and the noisy course of the contour lines indicating a quasi-random up and down of the DEM surface.

By analyzing the height spread (RMSE) of the dense throughwater DIM-derived 3D points within quadratic $25 \mathrm{~cm}$ cells as detailed in Section 2.2, a roughness map was derived, which helps to judge the DEM reliability. Especially in the submerged area, the waters' shear stress tends to smooth the bottom surface, and a 
high local RMSE value therefore indicates unreliable DEM parts. In the roughness map plotted in Figure 1d, the bright sandy areas (blue) can clearly be distinguished from both the submerged dark sea grass areas and the wooded slope (green, yellow, red). Blue hereby depicts areas with a local RMSE below $25 \mathrm{~cm}$. Keeping in mind that the RMSE was calculated from the 3D DIM-points potentially stemming from different stereo models, an RMSE in the range of $1-2 \mathrm{dm}$ (i.e. $<3$ pixels) also confirms good image orientation quality, which in this case was derived from direct georeferencing using GNSS/IMU data only.

It should be noted here that the RMSE values are even slightly lower in areas with higher image/stereo model coverage. Focusing on the central, reniform patch, the orthophoto (Figure 1b) exhibits a darker strip through the center of the patch marked with a yellow rectangle which is covered by two stereo models whereas the surrounding brighter areas contain points from three stereo models. The corresponding yellow rectangle area of Figure 1d exhibits slightly higher RMSE values caused by the lower redundancy $(\sqrt{n}$-law $)$.

Due to the lack of concurrently acquired LiDAR data, a horizontal water level was assumed for refraction correction of the raw DIM point clouds of Area I. The NIR channel of the UltraCam Osprey was hereby used to delineate the coastline and to estimate a mean water level.

\subsection{Area II}

In contrast to Area I, where no comparison data was available, the results of Area II (Poel, Baltic Sea) allow both a visual comparison of the underwater DIM results as well as a quantitative assessment. Figure 2a shows the colored DIM point cloud in ground plan view, highlighting the evident advantage of imagebased shallow water mapping, namely the availability of spectral information for each matched point. Figures $2 b$ and $c$ depict the DEMs derived from LiDAR and through-water DIM, respectively. The LiDAR derived DEM raster appears smooth and reliable to both sides of the water table, proving the high measurement quality of topo-bathymetric LiDAR data (Song et al., 2015). The DIM DEM, in turn, is much rougher in the submerged area. A good height correspondence is achieved at the small peninsula in the eastern part of the plotted area and in the near-shore shallow water areas. Especially the beach area at the western side of the peninsula shows a good DEM height agreement.

This can clearly be seen in the color coded DEM of Difference (DoD) model plotted in Figure 2d, from which the large, systematically positive height differences $(>2 \mathrm{~m})$ in the middle of the investigated area are standing out. Through-water DIM fails entirely at this sandy under water ridge with implausible heights above the water surface due to a lack of texture (smooth sand), further aggravated by small water surface riffles. Bathymetric LiDAR is much less affected in this case, as the laser footprint is typically larger than the small water waves present in this section of the scene and the LiDAR measurement does not depend on the availability of texture (i.e. radiometric differences). However, the DoD map also reveals that through-water dense matching works quite well in the adjacent deep part of the section with water depths of $>4 \mathrm{~m}$ (depth map, cf. Figure 2e). The sea floor is here covered with under water vegetation, which generates texture in the aerial images. A part of the height deviations in this area, visible in the color coded DoD map (Figure 2d) as small blue or red patches can therefore probably be explained by the up and down of the DIM surface between vegetation canopy and bottom surface, whereas only the bottom is mapped the LiDAR DEM. However, independent reference data would be necessary to answer this question reliably. While the DIM derived heights show a good agreement with the LiDAR data towards the western boundary of the study area by tendency, local differences in the $1 \mathrm{~m}$-range can still be observed. These differences might again be attributed to the existence of underwater vegetation, but DIM measurement errors due to the wavy water surface are a more likely explanation.

Figure $2 \mathrm{f}$ shows the color coded RMSE roughness map of the DIM dataset. This map allows a more quantitative interpretation of the dense matching results as, except at surface discontinuities, the influence of terrain surface roughness is negligible compared to the spread of the measured heights. The region of the peninsula and the adjacent shallow beach area show RMSE values well below $50 \mathrm{~cm}$ corresponding to the blue color tones in Figure $2 \mathrm{f}$. The red color, in contrast, indicates a local height spread of more than $2 \mathrm{~m}$, and therefore reveals areas where through-water dense matching is unfeasible for a high resolution description of submerged surfaces.

Even if the local precision measures are unfavorable for DIMbased underwater surface mapping in many places, the section view in Figure $2 \mathrm{~g}$ shows a good general trend between DIM and LiDAR derived heights, even in the deeper areas where one might expect inaccurate matching results due to the progressive blurring of submerged terrain features with increasing water depth. However, the general benefit of the higher spatial resolution of pixel-wise dense matching, depending mainly on the GSD of the imagery, compared to the relatively large laser footprint used in laser bathymetry is lost as large-scale smoothing of the underwater DIM point clouds is inevitable for obtaining acceptable results.

The quantitative comparison of Area II yields a precision (mean local RMSE) of $0.51 \mathrm{~m}$ for the through-water dense matching point cloud, and $0.05 \mathrm{~m}$ for the LiDAR dataset. Thus, for the entire Area II, the local spread of the height values is 10-times larger for DIM compared to the bathymetric LiDAR. The same calculation carried out for dry land (i.e. peninsula) only results in a RMSE of $0.15 \mathrm{~m}$ for DIM and $0.07 \mathrm{~m}$ for LiDAR, respectively, thus only a factor of two. The decline of precision in this open water scenario amounts to a factor of 5. Maas (2015) reports an accuracy decrease of a factor of 2 under laboratory conditions (planar water surface, clear water, etc. ) which is considered well in line with the reported precision achieved in this real-world data acquisition.

\subsection{Area III}

While the coastal datasets Area I (Borneo, Malaysia) and Area II (Baltic Sea, Germany) are comparable w.r.t. water clarity, water surface conditions (waves), availability of sea floor texture and, thus, yield similar results, the main problem with the Area III dataset (mountain lake) was turbidity. After a series of heavy thunderstorms in August 2017, the Secchi depth only amounted to $2.4 \mathrm{~m}$ when concurrently acquiring the RGB and CIR images and the topo-bathymetric LiDAR data on August, 22. Figure 3a and $b$ show a $\mathrm{RGB} / \mathrm{CIR}$ image pair depicting the area around mountain lake Blaue Lacke (elevation: ca. $2400 \mathrm{~m}$ above sea level). The RGB images were captured with a 39 MPix Hasselblad camera (focal length: $35 \mathrm{~mm}$ ). The flight was conducted in different altitudes starting with overview strips from an altitude of $1000 \mathrm{~m}$ a.g.l. corresponding to a GSD of $20 \mathrm{~cm}$ and gradually 

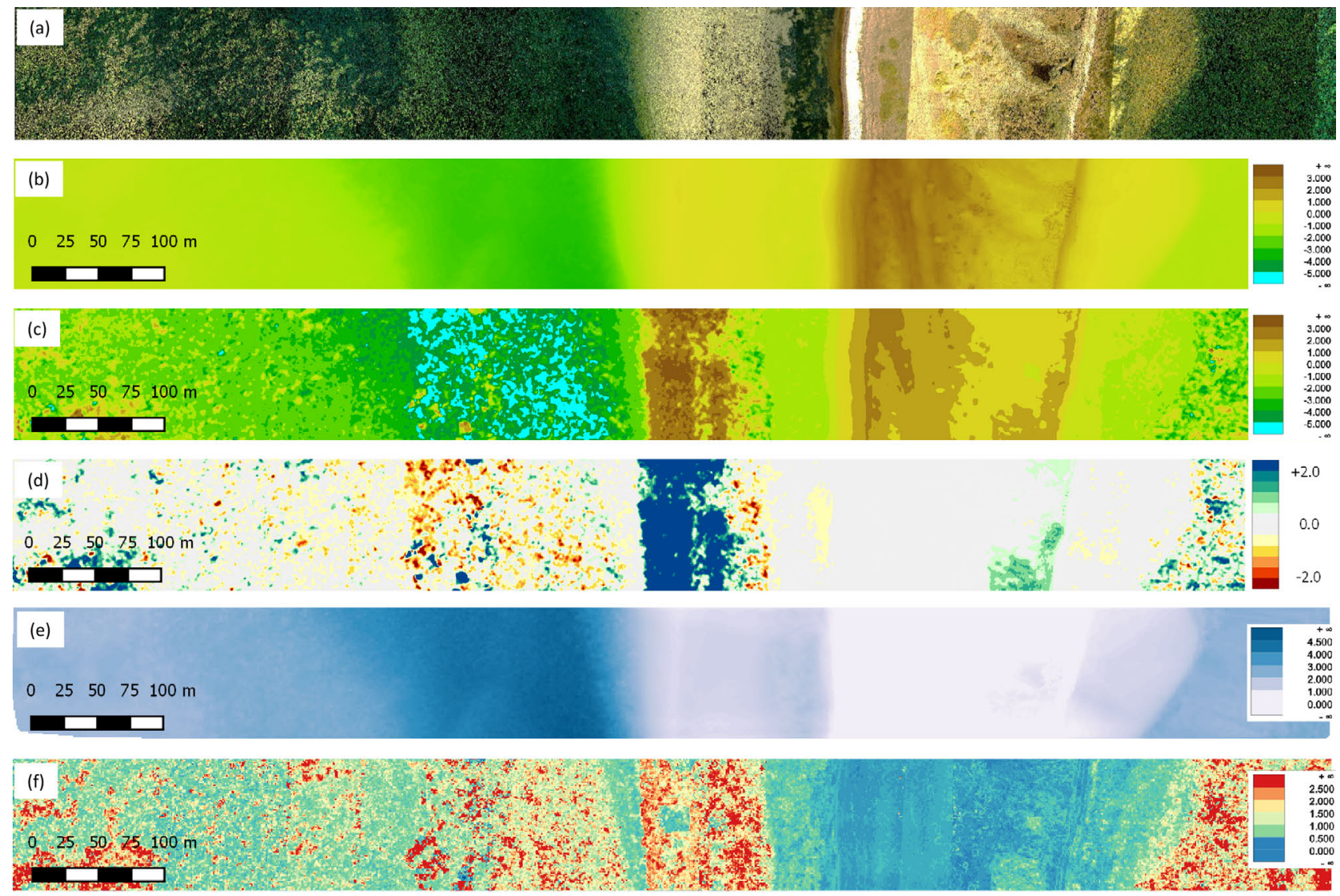

(g)

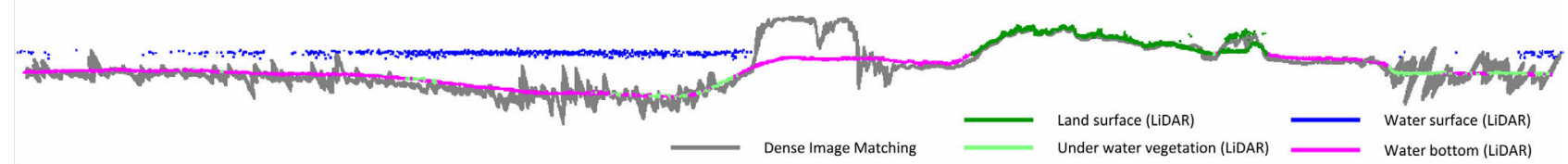

Figure 2. Results of Area II (Poel/German Baltic Sea); (a) plan view of colorized DIM point cloud; (b) LiDAR DEM, color coded elevation map; (c) DIM DEM, color coded elevation map; (d) Color coded DoD map (DIM minus LiDAR); (e) LiDAR derived water depth map; (f) RMSE of DIM point cloud (analysis unit: $25 \times 25 \mathrm{~cm}^{2}$ ); (g) DIM-LiDAR DEM profile comparison, location of section in the center of the Figure 2a-f, complete East-West-transect, heights exaggerated (max. depth: ca. $4 \mathrm{~m}$ )

lowering the altitude to finally $500 \mathrm{~m}$ a.g.1. (GSD: $10 \mathrm{~cm}$ ). While bundle block adjustment was carried out with Agisoft PhotoScan and Pix4D Mapper for the entire flight block, the RGB images from the lowest flying altitude were finally used for throughwater DIM. The simultaneously acquired CIR images (Tetracam, ADC Micro; cf. Figure 3b) were used for cross validation of the water-land boundary, which can be clearly identified in the infrared channel due to the high absorption of light at this wavelength. However, the water surface model itself, necessary for refraction correction of the raw DIM and LiDAR point clouds, was derived from the laser echoes using the statistical approach of Mandlburger et al. (2013). As the water surface of the mountain lake is very smooth with a height difference between inlet and outlet of approximately $15 \mathrm{~cm}$, the water level could be derived with $\mathrm{cm}$-precision by aggregating all near water surface laser echoes into $10 \mathrm{~m}$ raster cells.

While the lake bottom consisting of boulders with a diameter of $10-250 \mathrm{~cm}$ would have provided enough features for image matching, the unfavorable water turbidity conditions resulted in a loss of distinct image texture. Thus, through-water DIM was only successful in the shallow inlet area at the southern end of the lake. A perspective view of the RGB-colored DIM point cloud is plotted in Figure $3 \mathrm{c}^{1}$. Compared to the topo-bathymetric LiDAR point cloud, which consistently maps the entire bathymetry of the entire submerged inlet area (cf. Figure 3d), the DIM point represents the bottom of the lake only correctly in the very shallow area with water depths $<1 \mathrm{~m}$. In areas deeper than $1 \mathrm{~m}$, the quality and consistency of the image matching point cloud steeply declines with a chaotic behavior beyond a water depth of $2 \mathrm{~m}$.

The above mentioned correlation between DIM uncertainty and water depth can also be seen from the color coded DEM elevation maps (Figure $3 \mathrm{e}$ and $\mathrm{f}$ ). The superimposed $1 \mathrm{~m}$-contour lines show a satisfactory agreement in the dry littoral area of both the DIM and the LiDAR dataset. Beginning from the waterline (marked in Figure 3e), the DIM-DEM contour lines get fuzzier with increasing distance from the bank. The contour lines of the LiDAR DEM, in turn, show a consistent course also in the submerged area down to the penetration depth of approximately

\footnotetext{
${ }^{1}$ Remark: The black-and-white patch in the center of Figure $3 \mathrm{c}$ depicts a $3 \times 2 \mathrm{~m}^{2}$ large control patch laid out during the flight campaign for radiometric image calibration. Spectrally based remote sensing of bathymetry, however, is not the focus of the paper at hand.
} 

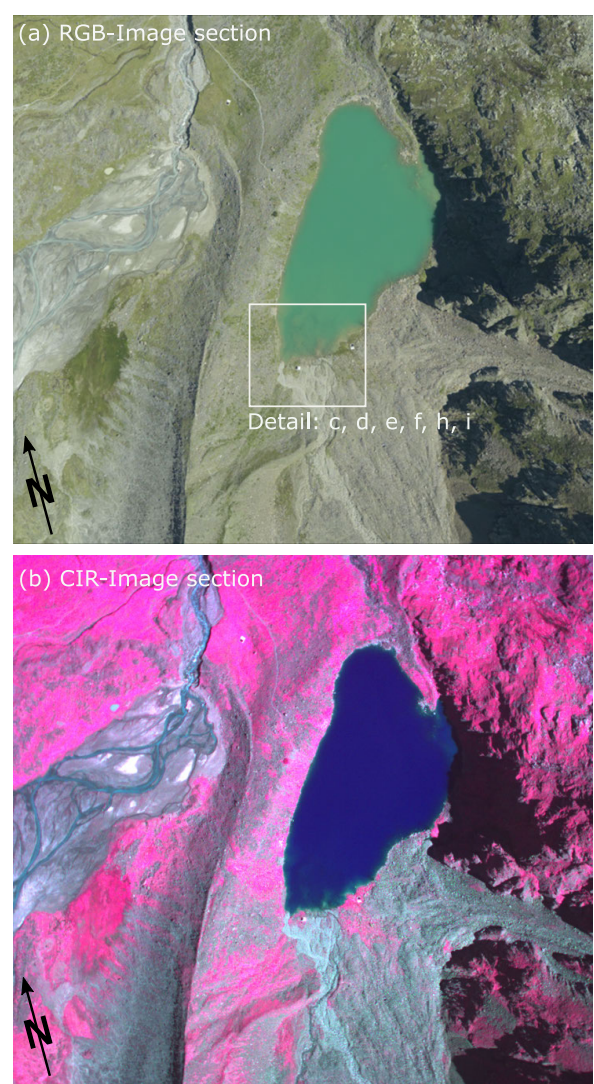

(i) DoD histogram (DIM minus LiDAR)
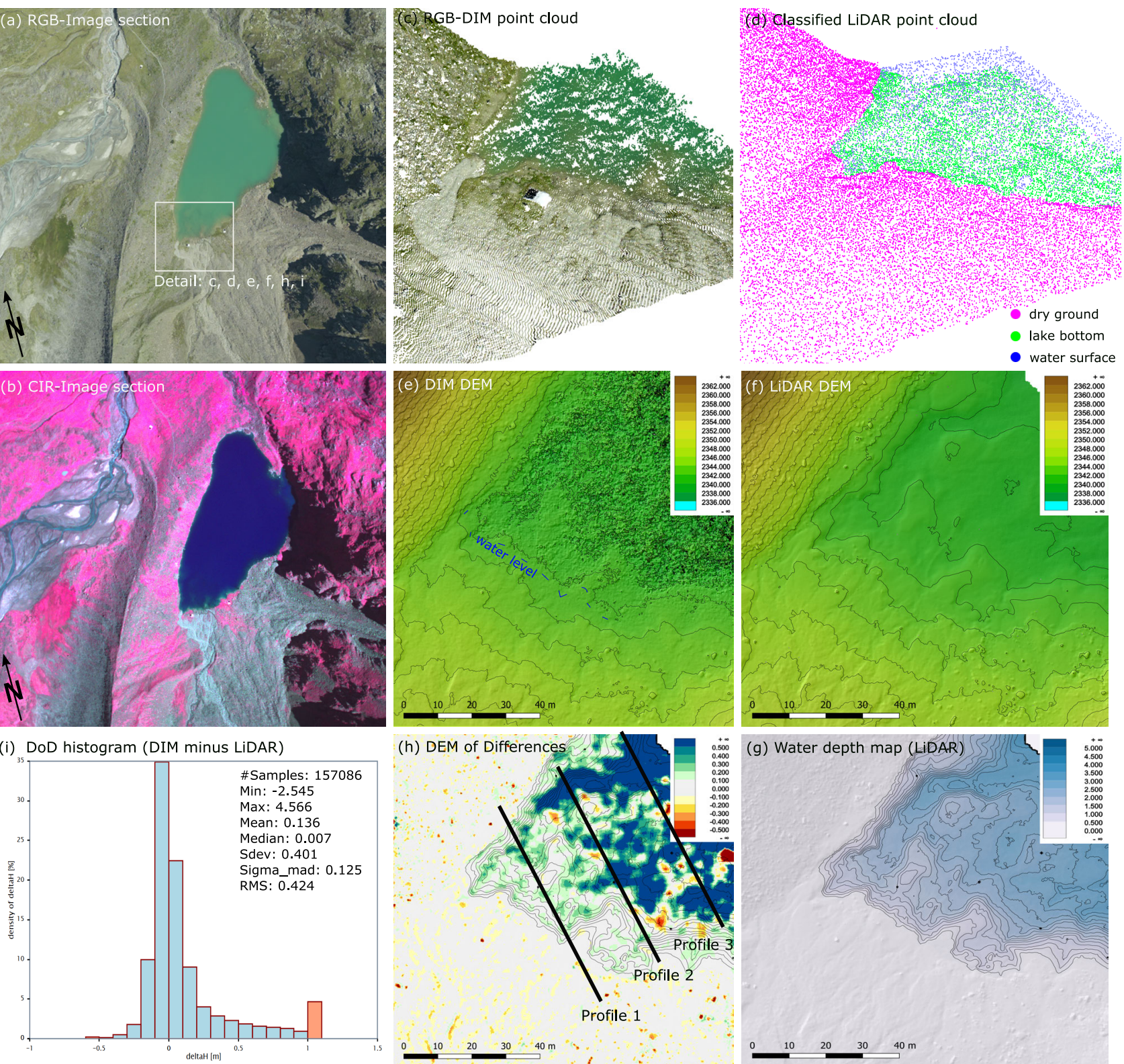

(h) DEM of Differences

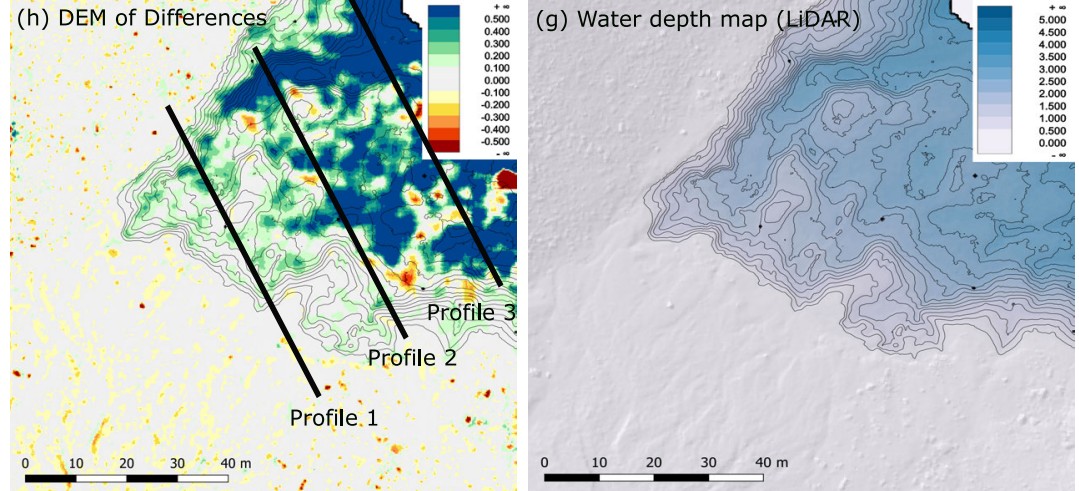

(j) Section view, Profile 1

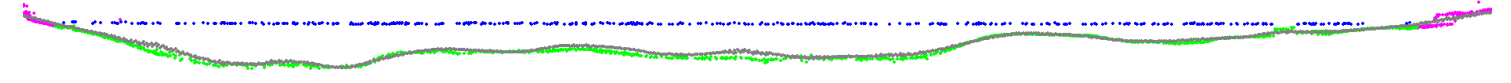

(k) Section view, Profile 2

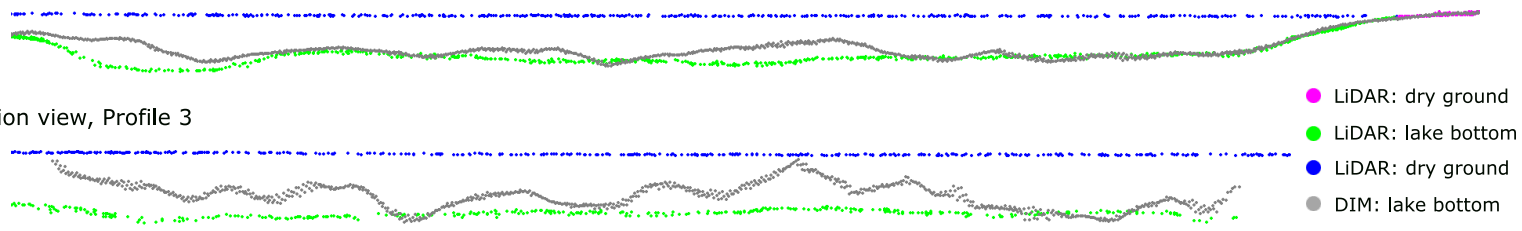

Figure 3. Results of Area III (Blaue Lacke, Stubai valley, Austria); (a/b) Section of original RGB-/CIR-image; (c) Perspective view of 3D DIM/LiDAR point cloud (DIM: RGB-colored, LiDAR: classified into ground, water bottom, water surface); (e/f) DIM/LiDAR DEM, grid spacing: $25 \mathrm{~cm}$, color coded elevation map superimposed with shaded relief map and $1 \mathrm{~m}$-contour lines; (g) LiDAR derived water depths superimposed with shaded relief; (h) Color coded DoD map (DIM minus LiDAR); (i) DoD histogram and basic statistics; (j-k) DIM-LiDAR DEM profile comparison, location of sections marked in (h), heights exaggerated (max. water depth: ca. $3.6 \mathrm{~m}$ )

$3.6 \mathrm{~m}$ (cf. depth map in Figure 3g.

Figure $3 \mathrm{~h}$ shows the deviations between the DIM- and the LiDAR
DEM. As already mentioned earlier, a good agreement between DIM and LiDAR with deviations between $-20-0 \mathrm{~cm}$ can be found outside the wetted perimeter indicated by the gray and light yel- 
low colors of the DoD map. In the submerged area, positive differences (green/blue) are dominating with occasional large negative (dark red) outliers as well. In general it can be said that the depth estimation is unreliable with deviations exceeding $\pm 50 \mathrm{~cm}$ in all but the most shallow areas. The vertical sections plotted in Figures 3j-1 finally underline and confirm the statements from above. Good height agreement can only be observed for Profile 1 (Figure 3j) but the height deviations reach unacceptable magnitude for Profile 3 (Figure 3j).

The DoD histogram of the entire scene is shown in Figure 3i allowing a detailed analysis of the DIM-LiDAR deviation distribution. The histogram shows a clear peak around zero and $55 \%$ of the deviations are less than $\pm 10 \mathrm{~cm}$ corresponding to the gray areas in Figure 3h. The highest contribution to this low-error class originates from the dry littoral area, which confirms the overall fitting precision of DIM and LiDAR derived point clouds in general and the quality of the image orientation in particular. On the left side, the histograms shows a steep descent (e.g., ca. $2 \%$ of the deviations are between $-30 \mathrm{~cm}$ and $-20 \mathrm{~cm}$ ). The occasional larger negative errors are mainly caused by the necessary low pass filtering of the DIM DEM, which was applied to achieve suitable results in the submerged area. This, in turn, causes undesirable smoothing of the larger convex boulders. The positive branch of the histogram descends much slower and the striking peak with $5 \%$ of the data exhibiting deviations of $>1 \mathrm{~m}$ stands out.

Therefore, Table 2 summarizes the depth dependent error metrics in detail. This table confirms the general increase of both the systematic error component (mean) as well as the dispersion (std.dev.) with increasing water depth. Especially the strictly positive bias $(11-31 \mathrm{~cm})$ is remarkable. The DIM-based water depth estimates are, thus, systematically underestimated for this dataset. If this is caused by the relatively high turbidity level gradually blurring the bottom features, has to be further investigated in future studies.

\begin{tabular}{rrr}
\hline Depth $[\mathrm{cm}]$ & Mean[cm] & Std.dev[cm] \\
\hline $0-50$ & 0.11 & 0.14 \\
$50-75$ & 0.20 & 0.19 \\
$75-150$ & 0.21 & 0.25 \\
$>100$ & 0.30 & 0.35 \\
\hline
\end{tabular}

Table 2. Water depth dependent error metrics

\section{CONCLUSIONS AND OUTLOOK}

In this contribution the general applicability of off-the-shelf dense image matching software for mapping shallow water bathymetry was evaluated. Respective tests were carried out at three different study areas comprising coastal areas (Area I: Borneo, Malaysia, Area II: Baltic Sea, Germany) and standing inland water bodies (mountain lake, Tyrol, Austria) using the software SURE (Rothermel et al., 2012; Wenzel et al., 2013), which implements a variant of the Semi-Global Matching (SGM) algorithm proposed by Hirschmuller (2008). In general, the feasibility of using through-water DIM for deriving shallow water bathymetry could be verified, but the resulting point clouds are less reliable compared to laser bathymetry, which is still the state-of-the-art for shallow water mapping.

Although further investigations including independent ground truth measurements are necessary to verify the findings of this first case study, the following main conclusions can be drawn:
- Through-water dense matching worked well given (i) clear water conditions, (ii) calm water surface, and (iii) availability of sufficient bottom texture. Whereas especially the latter is a general precondition for photogrammetry, the advent of DIM (Hirschmuller, 2008) has shown that pixel-wise height estimation is possible even in poorly textured areas due to the additional smoothness constraint within the SGM algorithm.

- Whereas the water bottom surface is often smooth as a result of the waters' shear stress, neighboring image pixels often show a substantial radiometric variation due the dynamic and wavy structure of the water surface causing refraction of the submerged part of the image ray in all possible direction. Thus, apparent parallaxes do not only occur in base direction but also perpendicular to the flight trajectory. For speeding up processing, the SURE software first rectifies stereo image pairs to epipolar geometry and subsequently performs dense matching only within the epipolar line. While this performance improvement is not part of the original SGM-approach (Hirschmuller, 2008), it is a commonly used DIM software technique (e.g. Trimble/Match$\mathrm{T})$. This, however, hampers feature matching if the image rays are refracted away from the epipolar line. Future investigations will concentrate on quantifying and compensating the effect of arbitrarily tilted water surfaces.

- In clear water water areas with sufficient bottom texture a good correspondence with the bathymetric LiDAR data was observed. An acceptable run of the DIM-based under-water surface, however, could only be achieved by extensive lowpass filtering of the original point cloud. This, in turn, decreases the effective spatial resolution considerably. With an applied filter kernel size of $1 \mathrm{~m}$, the spatial resolution of the DIM DEM is effectively lower than the achievable topobathymetric resolution. The latter is rather restricted by the footprint diameter of typically $50 \mathrm{~cm}$ than by the point density, which is often in the range of $5-20$ points $/ \mathrm{m}^{2}$.

- A quality decrease of the submerged DIM point cloud could be observed not only in case of missing bottom texture or undulating water surface, but also in case of rather turbid water conditions. Turbidity evidently reduces the contrast and poor texture directly influences the achievable accuracy. Especially for turbid water conditions (Area III) increasing deviations of the DIM-based heights compared to bathymetric LiDAR could be observed. This applies to both the bias $(10-30 \mathrm{~cm})$ and the standard deviation $(14-35 \mathrm{~cm})$.

- The conducted real-world experiments clearly showed that the submerged DIM point clouds and derived DEMs are acceptable in some areas but quickly become unreliable in case that at least one of the preconditions formulated above is not met. The study therefore also investigated methods to identify usable and unreliable areas, respectively. The local height spread (RMSE) has proven to be a good indicator.

To sum it up, the case study confirmed that through-water dense image matching is feasibly for mapping shallow water bathymetry under favorable conditions (clear water, calm water surface, bottom texture), but DIM suffers more from adverse environmental conditions (turbidity, wavy water surface, poor bottom texture) compared to bathymetric LiDAR. The latter profits from the fact of being an active, polar data acquisition technique. A single measurement is, thus, sufficient to record a $3 \mathrm{D}$ point 
if the signal strength of the laser echo reflected from the water bottom is strong enough. Apart from this, off-the-shelf DIM software relies on the collinearity equation in general and uses epipolar geometry in particular, but does not consider bending of the image rays due to refraction at the air-water interface. Whereas a general compensation of the apparent light rays was carried out in post-processing after image matching (Wimmer, 2016), light refraction also deteriorates the matching results in the first place. Future work on subject matters will therefore focus on potential corrections in the image space before stereo matching rather than correcting the raw DIM point clouds in post-processing in object space. Good prospects of success for such an approach are expected for concurrently acquired LiDAR and multi-spectral image data.

\section{ACKNOWLEDGEMENTS}

The presented study was conducted within the project 'Bathymetry by fusion of airborne laser scanning and multispectral aerial imagery' funded by the German Research Foundation (DFG). The author wants to thank Michael Vetter (Leica Geosystems) and Philipp Hüttl (nFrames) for providing software support and Michael Wimmer (GEO/TU Vienna) for providing the MATLAB code of the refraction correction tool. Furthermore, the author gives thanks to Bundesamt fr Seeschifffahrt und Hydrographie (BSH), Vexcel Imaging, Graz, and Amt der Tiroler Landesregierung for providing the image data of Area I, II and III.

\section{References}

Birkebak, M., Eren, F., Peeri, S. and Weston, N., 2018. The effect of surface waves on airborne lidar bathymetry (alb) measurement uncertainties. Remote Sensing 10(3), pp. 453.

Glira, P., Pfeifer, N., Briese, C. and Ressl, C., 2015. A Correspondence Framework for ALS Strip Adjustments based on Variants of the ICP Algorithm. PFG Photogrammetrie, Fernerkundung, Geoinformation 2015(4), pp. 275-289.

Guenther, G. C., Cunningham, A. G., Laroque, P. E. and Reid, D. J., 2000. Meeting the accuracy challenge in airborne lidar bathymetry. In: Proceedings of the 20th EARSeL Symposium: Workshop on Lidar Remote Sensing of Land and Sea, Dresden, Germany.

Hirschmuller, H., 2008. Stereo Processing by Semiglobal Matching and Mutual Information. IEEE Trans. Pattern Anal. Mach. Intell. 30(2), pp. 328-341.

IHO, 2008. S-44, Standards for Hydrographic Surveys. Standard 5th ed., International Hydrographic Organization, Monaco. http://www.iho.int/iho_pubs/standard/S-44_5E.pdf.

Legleiter, C. J., 2016. Inferring river bathymetry via ImagetoDepth Quantile Transformation (IDQT). Water Resources Research 52(5), pp. 3722-3741.

Lyzenga, D. R., Malinas, N. P. and Tanis, F. J., 2006. Multispectral bathymetry using a simple physically based algorithm. IEEE Transactions on Geoscience and Remote Sensing 44(8), pp. 2251-2259.

Maas, H.-G., 2015. On the Accuracy Potential in Underwater/Multimedia Photogrammetry. Sensors 15(8), pp. 1814018152.
Mandlburger, G., Hauer, C., Wieser, M. and Pfeifer, N., 2015. Topo-bathymetric lidar for monitoring river morphodynamics and instream habitats-a case study at the pielach river. Remote Sensing 7(5), pp. 6160.

Mandlburger, G., Pfennigbauer, M. and Pfeifer, N., 2013. Analyzing near water surface penetration in laser bathymetry a case study at the river Pielach. In: ISPRS Annals of the Photogrammetry, Remote Sensing and Spatial Information Sciences, Vol. II-5/W2, pp. 175-180.

Mandlburger, G., Wenzel, K., Spitzer, A., Haala, N., Glira, P. and Pfeifer, N., 2017. Improved topographic models via concurrent airborne lidar and dense image matching. ISPRS Annals of Photogrammetry, Remote Sensing and Spatial Information Sciences IV-2/W4, pp. 259-266.

Pfeifer, N., Mandlburger, G., Otepka, J. and Karel, W., 2014. Opals a framework for airborne laser scanning data analysis. Computers, Environment and Urban Systems 45, pp. 125 -136 .

Rothermel, M., Wenzel, K., Fritsch, D. and Haala, N., 2012. SURE: Photogrammetric surface reconstruction from imagery. In: Proceedings of the Low Cost 3D Workshop, Berlin.

Rupnik, E., Jansa, J. and Pfeifer, N., 2015. Sinusoidal wave estimation using photogrammetry and short video sequences. Sensors 15(12), pp. 30784-30809.

Schwarz, R., Pfeifer, N., Pfennigbauer, M. and Ullrich, A., 2017. Exponential decomposition with implicit deconvolution of lidar backscatter from the water column. PFG - Journal of Photogrammetry, Remote Sensing and Geoinformation Science 85(3), pp. 159-167.

Song, Y., Niemeyer, J., Ellmer, W., Soergel, U. and Heipke, C., 2015. Comparison of three airborne laser bathymetry data sets for monitoring the german baltic sea coast.

Wenzel, K., Rothermel, M., Haala, N. and Fritsch, D., 2013. SURE the ifp Software for Dense Image Matching. In: D. Fritsch (ed.), Photogrammetric Week '13, Wichmann/VDE Verlag, Berlin \& Offenbach, pp. 59-70.

Westaway, R., Lane, S. and Hicks, D., 2001. Remote sensing of clear-water, shallow, gravel-bed rivers using digital photogrammetry. Photogrammetric Engineering and Remote Sensing 67(11), pp. 1271-1281.

Wimmer, M., 2016. Comparison of active and passive optical methods for mapping river bathymetry. Master's thesis, Department für Geodäsie und Geoinformation. https://resolver.obvsg.at/urn:nbn:at:at-ubtuw:1-2619. 